\title{
The role of ITS in evacuation route optimization for emergency vehicles
}

\author{
A. Polimeni \& A. Vitetta \\ DIMET, Dipartimento di Informatica, Matematica, Elettronica e \\ Trasporti,Università degli Studi Mediterranea di Reggio Calabria, Italy
}

\begin{abstract}
In this paper the application of ITS (Intelligent Transportation Systems) in road evacuation in the case of route optimization for emergency vehicles is reported. The ITS are usually used in transport to analyse the data and improve the system performances. In road evacuation, the ITS can be used at three levels: survey and transmission, control and user information. This structure allows managing an evacuation starting from the network monitoring and defining the optimal strategies to apply to speed up the evacuation procedure.
\end{abstract}

Keywords: vehicle routing; link cost functions; network monitoring.

\section{Introduction}

The road evacuation researches involve different fields of study, concerning demand analysis [1-5], demand-supply interaction [6], route design [7] and planning [8-12]. For a study in depth, see [13].

ITS (Intelligent Transportation Systems) are a set of hardware and software systems who allows collecting, processing and distributing some information to improve the travel of people and freight [14].

In many countries a national architecture for ITS system is implemented, to improve the transport systems (i.e. in Italy is named ARTIST). The emergency vehicles management is provided in the national architectures (i.e. the emergency management system in Italy or the emergency management in U.S.). The ITS assume a core role in road evacuation, because they allow to manage the information in real time and hence to address the evacuees. Using real-time information allows defining a travel plan for users to reduce the total evacuation time [15]. 
In the following (Figure 1), three main classes of ITS are considered: ITS for survey and data transmission, ITS for control and ITS for user information.

The first class (survey and transmission) allows surveying the data (i.e. flow) and transmitting it. The second class (control) allows to processing the data and, using appropriate tools, to establish the strategies to optimize the objectives. The third class (user information) allows sending the strategies at the users (private users and rescue vehicles). The paper is structured so that explicates these three

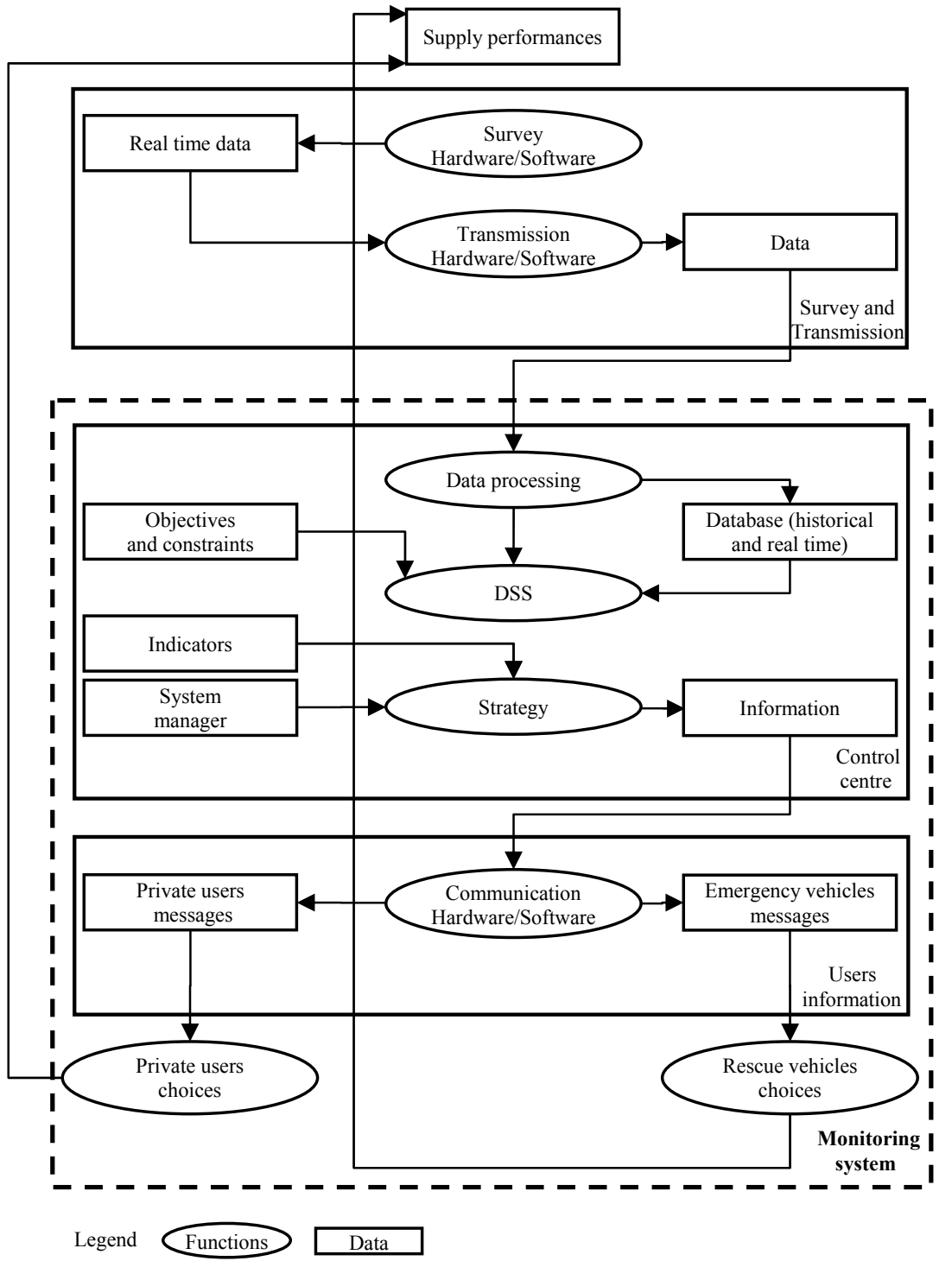

Figure 1: $\quad$ ITS for monitoring and management. 
classes in relation to route optimization. Section 2 deepens the data survey and transmission; Section 3 analyses the control system; Section 4 reports the methodologies to send the messages at the users. Finally, in Section 5 some conclusions are reported.

\section{Data survey and transmission}

The road network survey can be crucial in many cases, from capturing the network actual status to capturing the network modifications. The collected data can be used to operate in real time (i.e. disseminating the information to the users) or to forecast the network response due the external events (i.e. to individuate possible critical points). The needs are related to monitoring in real time the traffic conditions evaluating (flow, velocity, travel time). In Table 1 the user needs and the possible functions able to satisfy them are reported.

Table 1: $\quad$ Data survey and transmission: user needs and functions.

\begin{tabular}{cll}
\hline Needs & \multicolumn{1}{c}{ Functions } \\
\hline \multirow{3}{*}{ Survey and transmission } & $\bullet$ & Collect traffic data \\
& $\bullet$ & Monitor network vehicle occupation \\
& $\bullet$ & Infrastructure monitor \\
\hline
\end{tabular}

The data survey can be made manually or automatically $[17,18]$. In the first case, operators on the road survey some flow characteristics (i.e. number of vehicles, vehicle type, velocity) who can be analysed at a later stage. This method can be lack related to operator necessity. Conversely, the automated means allows surveying the flow characteristics in conditions not favourable for human operators. For example, during a road evacuation, an automated approach can be used to survey specific data related to situation (damaged roads, congested links and so on).

The data survey is based on a hardware/software system: the hardware is a sensor or a network sensor (i.e. a camera, radar, infrared systems, GPS) that monitor the traffic, the software decode the signal sent by the sensors and save the data. According to [17], can be distinguish tripwire sensors, who operate in a road section (like a camera), and tracking sensors, who are able monitoring an area or track vehicles trajectories. An example of vehicle tracking is reported in [19], in which this system is used to optimize a bus line. In some cases, probe vehicles can be used to measure the network characteristics [20]. Other approaches [21] consider sensors for short duration survey and sensors for permanent survey for collecting traffic load data.

Also the transmission system is composed by hardware/software tools able to manage the data transmission. Two ways are possible: transmission on-line and transmission off-line. In first case, the data are sent to the command centre using some technologies, as GSM, internet, wireless and so on. In second case, the data are stored in a fixed support (connected to the sensors) that must be carried by an operator to the control centre. 
In literature, some works investigate this field. In [22] some technologies to survey a road network to measure the travel time are reported. Marzolf et al. [23] propose an object-oriented approach to survey the system using a probe vehicle equipped with a GPS. The aim is allowing at the vehicle to visit all links. A network sensor location problem is solved in [24] to identify subsets of links in which locate the sensors to survey flow data. An approach using some cameras to survey the network is reported in [25]. ITS technology was also used post a sudden event happening to analyse the damage incurred in the network [26]. In [27] an emergency management information system is reported; this system allows the data communication between the control centre and the operators involved in the emergency. Also in routing problem the data survey in real time play a fundamental role, in freight transport in [28] and [29] real time information on link travel time are provided by a probe vehicle equipped with a personal digital assistant and GPS antenna and used to improve vehicle routes. Considering rescue vehicles, the vehicle management is indicated with Computer Aided Dispatching (CAD). In [30] an integrated system for ambulances management is described, a part of this system is a GSM modem able to transmit the vehicle position (and eventually vocal messages) at the control centre. In Figure 2 a schematic representation of means related to data survey and transmission is reported, summarizing the considerations reported in this section. Some means able to collect traffic data are considered. Considering the automatic survey, in the tripwire sensors can be considered loop sensors, video sensors, optical or acoustic sensors. In some cases, these instruments can be used joint to refine the collected data. About the transmission, the off-line and on-line possibilities are considered; in the first case an operator is need to retrieve data, in the second case the data are sent automatically.

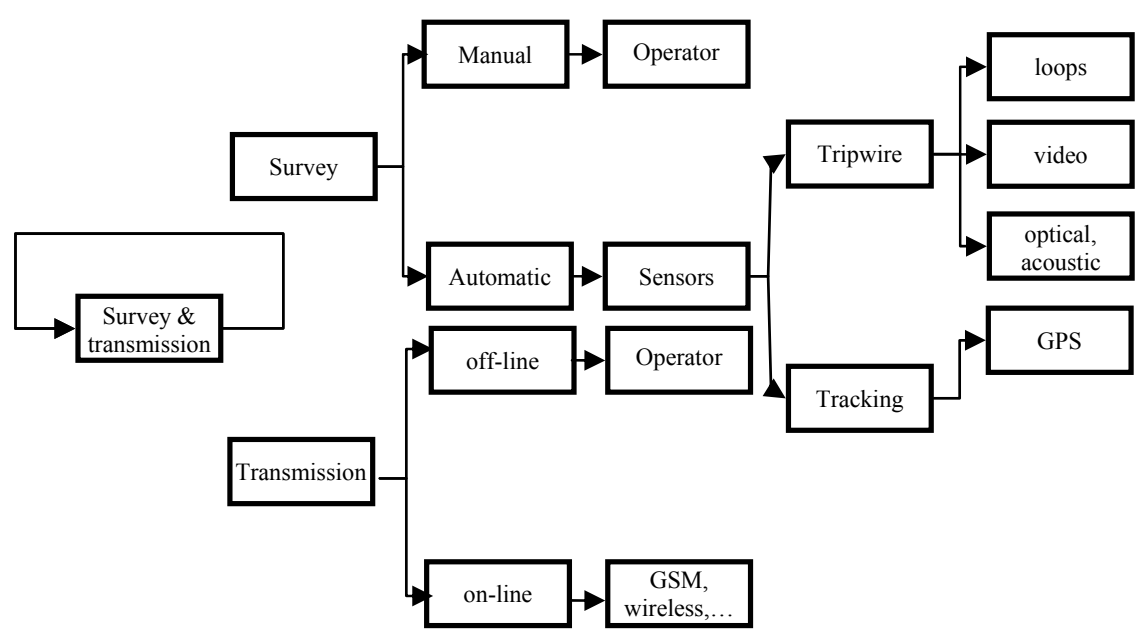

Figure 2: $\quad$ ITS for monitoring and management. 


\section{Data processing}

In literature, the use of various Decision Support Systems (DSSs) to simulate an evacuation and to optimize the best strategies to apply in an evacuation is proposed. An inventory of these DSSs is reported in [31]; an example in the use of these DSSs is reported in [32]. When the data are transmitted at the command centre, a control procedure is needed to elaborate the data and define the strategies to apply to face the situation. In a first stage, the data can be filtered to eliminate implausible records and given as input to a DSS who allows to define the optimal strategies (in respect to objectives and constraints) to put in action. The DSS can be use also historical data in the strategies definition. In Table 2 the user needs and the possible related function are reported. General considerations on DSS use in disaster management are reported in [33].

Table 2: $\quad$ Data processing: user needs and functions.

\begin{tabular}{cll}
\hline Needs & & \multicolumn{1}{c}{ Functions } \\
\hline & $\bullet$ & Evaluate Trip \\
Data processing & $\bullet$ & Implement Trip Plan \\
& $\bullet$ & Provide Traffic Control \\
& $\bullet$ & Provide Traffic Forecasts and Strategies \\
& Provide Traffic Management \\
\hline
\end{tabular}

The DSS can be specific for a single aspect of the problem (in this case several DSS can be used to solve the whole problem), or general to consider simultaneously all the aspects of the problem. In first case, for example, can be consider a DSS to evaluate the network performances and a DSS to optimize the paths. In second case, the DSS is able to analyse the system and define the strategies to apply [34]. Further considerations can be made considering that, in some cases, using historical data, is possible a pre-processing procedure, for example to allocate resource on the network [35] to speed up the operations during the evacuation. In the field of route optimization, many DSS can be considered to optimize (or re- optimize) the routes for emergency vehicles. In this case, the output is a route (or a set of route) to assign at the rescue vehicles minimizing an objective (minimize evacuation time). In [36] a tool to manage in real time an evacuation plan is proposed. This tool, given the evacuation area, allows optimizing the evacuation paths for the users using a heuristic procedure. Tufekci [37] proposes a modular DSS to hurricane evacuation management. The modules composing the DSS have a specific function, like prediction and tracking, planning, emergency management, coordination; moreover a GIS module is implemented to furnish graphical information. In [38] a DSS named Group Analytic Network Process (GANP) is proposed. This is a DSS allowing decision-makers to compare some alternatives in a multi-criteria approach. In [39] an open DSS (EPLAN) able to integrate data from different sources, to perform data analysis and model computation is provided. In Figure 3 a schematic representation of data processing in reported, considering DSS able to 
solve a specific part of the problem and DSS able to solve the whole problem. In first case, a DSS can be used to get, for example, the network performances (network optimization) and another can be used for route optimization. In second case, an only DSS can be used for solving simultaneously network and route optimization.

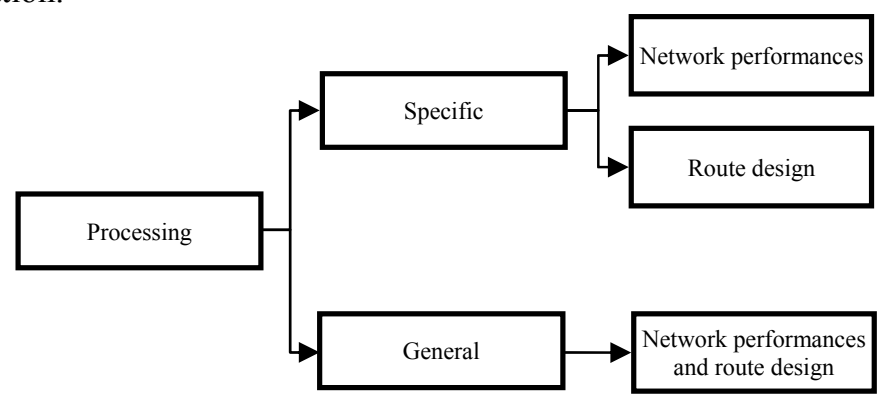

Figure 3: $\quad$ ITS for monitoring and management.

\section{User information}

Once acquired and processed by the DSS, the data must be sent to the users. Two cases can distinguish, considering if the users are the rescue operators or the people who evacuate. In first case, command centre use information to coordinate the rescue operations. In second case, the information are sent directly to the population suggesting or imposing the actions to follow.

An efficient method need to send the information as soon as possible also in emergency conditions. Various communication systems can be used (radio, telephone, mobile, internet, SMS, GSM, wifi and so on). The sending of information to the users is investigate in some papers, for example, in [25] and [40] an analysis on the effects related to the data sending with variable message panels is proposed; in [41] an analysis to determine what data to send and how send it is reported; in [42] user behaviour by sending travel information is analysed. In most case, these communication systems are indicated as Advanced Traveler Information System (ATIS [43]). The information sent, in general, change the user behaviour, like in term of path choice $[44,45]$ and consequently change in traffic assignment [46]. In Table 3 the user needs and the possible related function for data communication are reported.

Table 3: $\quad$ Data communication: user needs and functions

\begin{tabular}{cll}
\hline Needs & \multicolumn{1}{c}{ Functions } \\
\hline & $\bullet$ & Plan trip \\
Data communication & $\bullet$ & Propose trip alternatives \\
& $\bullet$ & Support trip \\
& $\bullet$ & Assess perturbations \\
\hline
\end{tabular}


The network monitoring systems provides real-time traffic conditions and, consequently, the information can be address the users towards other paths. In fact, the travel information modifies the user's behaviour, also according to how information is sent [47]. The evacuation managers, to clearing the path for rescue vehicles can be using this method [48]. In [36], a system to address the user in the networks is proposed; this system is based on light signals located at nodes to indicate at the users the path to follow.

A module of the system proposed in [37] is reserved to the communications (satellite connection), able to operate in emergency conditions when the normal communication channels can fail.

In logistic field, to optimize the vehicles route and the vehicle allocation, CVRS (Computerised Vehicle Routing and Scheduling) systems can be used, this system can give information at the vehicles (i.e. users' position). In emergency field, the vehicle dispatching, starting from data available (i.e. user position, user condition, ...) can be managed using CAD systems able, among other things, to send radio messages at the vehicle (ambulances, fire trucks) [49]. In [30] GSM network communication is used to send messages to the ambulances, these messages are related to the user position, path to following. Furthermore, the ambulance can be equipped with a monitor to display the path calculate by the control centre. In the MAGES (Mature Applications of Galileo for Emergency Scenario) project [50] the support to operations for the management of various emergency types was expected. In [50] an example of MAGES application is explained: it is possible to communicate the target and the path to reach him, integrating traffic information.

Figure 4 summarize, in a schematic way, the sending of information at the users, using some possible communication systems. The information provided are related to the netwok conditions (i.e. closing links), paths to follow, users positions (i.e. to communicate in real time new users to rescue).

During an emergency, the communication systems can be fail for various reasons (i.e. damage to the physical elements of the system, system overload,

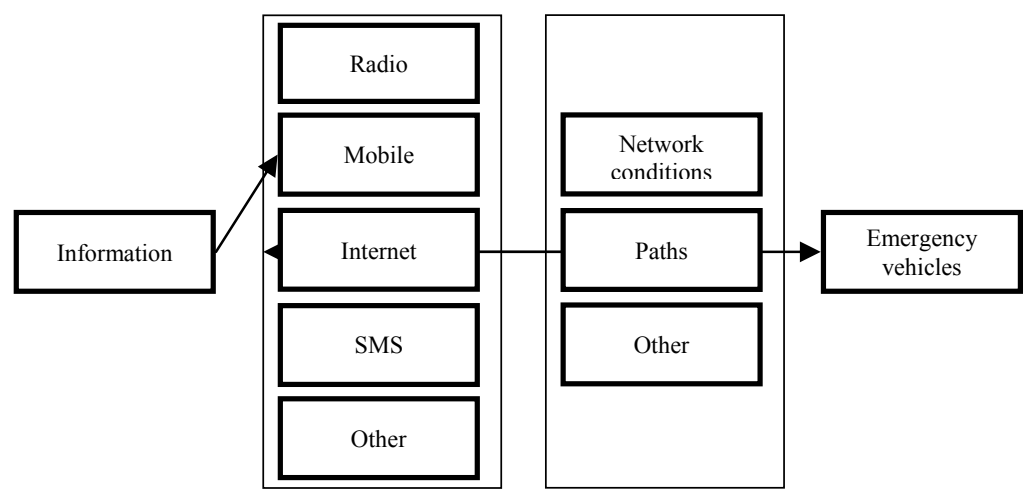

Figure 4: Sending information at the emergency vehicles. 
Table 4: Data communication: user needs and functions.

\begin{tabular}{cc}
\hline ITS & Reliability \\
\hline radio & medium \\
telephone & low \\
mobile & medium \\
internet & medium \\
SMS & medium \\
wifi & low \\
satellite & high \\
\hline
\end{tabular}

and so on). It is necessary to evaluate the reliability of the available communication systems. In Table 4 a qualitative reliability analysis is reported.

\section{Experimentation}

A prototype of a system to collect data from a real network is designed. In this prototype, the data are collected using an hardware/software system able to record some travel information. According to the definition given in the previous sections, the survey system is automatic with a tracking sensor while the transmission system is off-line, managed by an operator.

The real case application is designed to detect the paths and stops of a rescue vehicle. The ITS used is PDA equipped with GPS sensor and a software able to save the position at fixed instant. The operators can be to interact intuitively with the system to save additional positions (i.e. for rescue vehicles, the positions in which they intervene) and additional information (i.e. if the rescue vehicle is an ambulance, the number of users rescued). The analyst can obtain more information (travel time, duration of the stops) during the data analysis. In Table 5 some possible data obtainable with the experimentation are reported.

Table 5: Data communication: user needs and functions.

\begin{tabular}{|c|c|c|c|c|c|c|c|c|c|}
\hline ID & UTC & Latitude & & Longitud & & Cause $^{*}$ & $\begin{array}{c}\text { Duration } \\
\text { (sec.) }\end{array}$ & Number of users & Notes \\
\hline 1 & 70248 & 37.92070 & $\mathrm{~N}$ & 15.78355 & $E$ & $\mathrm{~A}$ & 348 & 2 & - \\
\hline 2 & 81281 & 37.92134 & $\mathrm{~N}$ & 15.77176 & E & B & 512 & - & Medical \\
\hline 3 & 86147 & 37.92131 & $\mathrm{~N}$ & 15.77851 & $\mathrm{E}$ & $\mathrm{C}$ & 155 & - & Technical stop \\
\hline.. & .. & .. &.. & .. & .. & .. & .. & .. & .. \\
\hline
\end{tabular}

* A: users rescue, B: supplies delivery, C: other.

Starting from the available data collected by the survey systems, the operative centre elaborate its (also using historical data) and can simulate a sudden event that forces an evacuation. In this way it is possible to optimize in advance the paths for users and rescue vehicles, and for example establish the means to furnish information to deviate the demand flow. Moreover, it is possible to establish contraflow strategies to reduce evacuation time and design alternative paths. 


\section{Conclusions}

In this paper a discussion on use of ITS in road evacuation is conducted. Three main classes of ITS are examined: ITS for survey and transmission, control and user information. For each class, the main use of ITS is analysed.

The data survey and transmission is analysed considering the possible means to collect the data and send it at a control centre. The aim of the control is the data analysis to elaborate the strategies to apply during an evacuation. The strategies need to be sent at the user: the communication system is analysed considering the possible means.

Finally, a short experimentation is reported.

\section{Acknowledgement}

This research is partially supported by national MIUR under PRIN2009 grants n. 2009EP3S42_001.

\section{References}

[1] Russo F. and Chilà G., Safety of users in road evacuation: demand models. WIT Transactions on the Built Environment, Volume 96, Urban Transport XIII, Urban Transport and the Environment in the 21st century, Brebbia C. A. (ed.), WIT Press, Southampton, pp. 773-782, 2007.

[2] Russo F. and Chilà G., Safety of users in road evacuation: RP vs. SP surveys in demand analysis. WIT Transactions on the Built Environment, Volume 101, Urban Transport XIV, Urban Transport and the Environment in the 21st century, Brebbia C. A. (ed.), WIT Press, Southampton, pp. 703713, 2008.

[3] Russo F. and Chilà G., Safety of users in road evacuation: Modelling and DSS for demand. WIT Transactions on Ecology and the Environment, Volume 120, Sustainable Development and Planning IV, vol.1, Brebbia C. A. (ed.), WIT Press, Southampton, pp. 465-474, 2009.

[4] Russo F. and Chilà G., Demand models in road evacuation: a synopsis of recent contributions. WIT Transactions on the Built Environment, Volume 116, Urban Transport XVII, Urban Transport and the Environment in 21st Century, Pratelli \& Brebbia C. A. (ed.), WIT Press, Southampton, pp. 601614, 2011.

[5] Russo F. and Chilà G., A statistical approach to analyse user behaviour in road evacuation. WIT Transactions on the Built Environment, Volume 117, Safety and Security engineering $I V$, Guarascio M., Reiners G. Brebbia C. A. \& Garzia F. (ed.), WIT Press, Southampton, pp. 377-390, 2011.

[6] Vitetta A., Musolino G. and Marcianò F.A., Safety of users in road evacuation: Supply and demand-supply interaction models for users. WIT Transactions on the Built Environment, Volume 96, Urban Transport XIII, Urban Transport and the Environment in the 21st century, Brebbia C. A. (ed.), WIT Press, Southampton, pp. 783-792, 2007. 
[7] Vitetta A., Quattrone A. and Polimeni A., Safety of users in road evacuation: design of path choice models for emergency vehicles. WIT Transactions on the Built Environment, Volume 96, Urban Transport XIII, Urban Transport and the Environment in the 21st century, Brebbia C. A. (ed.), WIT Press, Southampton, pp. 803-812, 2007.

[8] Russo F. and Rindone C., Safety of users in road evacuation: planning internal processes and guidelines. WIT Transactions on the Built Environment, Volume 96, Urban Transport XIII, Urban Transport and the Environment in the 21st century, Brebbia C. A. (ed.), WIT Press, Southampton, pp. 825-834, 2007.

[9] Russo F. and Rindone C., Safety of users in road evacuation: the logical framework approach in evacuation planning. WIT Transactions on the Built Environment, Volume 101, Urban Transport XIV, Urban Transport and the Environment in the 21 st century, Brebbia C. A. (ed.), WIT Press, Southampton, pp. 751-760, 2008.

[10] Russo F. and Rindone C., Safety of users in road evacuation: Modelling and DSS for LFA in the planning process. WIT Transactions on Ecology and the Environment, Volume 120, Sustainable Development and Planning IV, vol.1, Brebbia C. A. (ed.), WIT Press, Southampton, pp. 453-464, 2009.

[11] Russo, F. and Rindone C ., The planning process and logical framework approach in road evacuation: a coherent vision. WIT Transactions on the Built Environment, Volume 117, Safety and Security engineering IV, Guarascio M., Reiners G. Brebbia C. A. \& Garzia F. (ed.), WIT Press, Southampton, pp. 415-425, 2011.

[12] Russo, F. and Rindone C ., Planning in road evacuation: classification of exogenous activities. WIT Transactions on the Built Environment, Volume 116, Urban Transport XVII, Urban Transport and the Environment in $21^{\text {st }}$ Century, Pratelli \& Brebbia C. A. (ed.), WIT Press, Southampton, pp. 639651, 2011.

[13] Russo F. and Vitetta A., Risk evaluation in a transportation system. International Journal of Sustainable Development and Planning, 1 (2), pp. 170-191, 2006.

[14] Russo F. and Quattrone A., ITS - Sistemi di Trasporto Intelligenti. Elementi di base e applicazioni operative per il trasporto privato, per il trasporto pubblico, per il trasporto merci e la logistica. FrancoAngeli, Milano, 2010.

[15] Roldão B., Intelligent Transport Systems on Emergency Evacuation Modelling. In Proc. of $12^{\text {th }}$ WCTR, 2010. Source: http://intranet.imet.gr /Portals/0/UsefulDocuments/documents/02622.pdf. (Last access: 12/01/12).

[16] Architettura Telematica Italiana per il Sistema dei Trasporti, http://www.its-artist.rupa.it/index.htm (Last access: 13/12/11).

[17] Iera A., Modafferi A., Musolino G. and Vitetta A., An experimental station for real-time traffic monitoring on a urban road. IEEE $5^{\text {th }}$ international conference on intelligent transportation systems, pp. 697-701, 2007. 
[18] Chirico P. and Vitetta A., Un sistema di monitoraggio del traffico veicolare: tecniche di rilievo dei parametri di traffico ad analisi dei dati. Metodi e Tecnologie dell'Ingegneria dei Trasporti, FrancoAngeli, Milano, pp. 332346, 2000.

[19] Balbo F. and Pinson S., Using intelligent agents for transportation regulation support system design. Transportation Research C, 18, pp. 140$156,2010$.

[20] Hellinga B., Izadpanah P., Takada H. and Fu L., Decomposing travel times measured by probe-based traffic monitoring systems to individual road segments. Transportation Research C, 16, pp. 768-782, 2008.

[21] NCHRP, Report 5009, Equipment for collecting traffic load data, Transportation Research Board, 2004.

[22] Jie L., van Zuylen H., Chunua L. and Shoufeng L., monitoring travel time in an urban network using video, GPS and Bluetooth. Procedia Social and Behavioural Science, 20, pp. 630-637, 2011.

[23] Marzolf F., Trépanier M. and Langevin A., Road network monitoring: algorithms and a case study. Computers and operations research, 33, pp. 3494-3507, 2006.

[24] Hu S.H., Peeta S., Chu C.H., Identification of vehicle sensor location for link based network traffic application. Transportation Research B, 43, pp. 873-894, 2009.

[25] Lam W. H. K. and Chan K. S., A model for assessing the effects of dynamic travel time information via variable message signs. Transportation, 28, pp. 79-99, 2001.

[26] Bono F. and Gutiérrez E., A network based analysis of the impact of structural damage on urban accessibility following a disaster: the case of the seismically damaged Port Au Prince and Carrefour urban road networks. Journal of Transportation Geography, 19, pp. 1443-1455, 2011.

[27] Atteih A.S., AlQahtani S.A. and Nazmy A., Emergency management information system: case study. Source: http://www.saudigis.org/ FCKFiles/File/SaudiGISArchive/4thGIS/Papers/15_SalmanQahtani_ KSA.pdf (Last access: 16/01/2012).

[28] Ando N. and Taniguchi E., Travel time reliability in vehicle routing and scheduling with time windows. Networks and Spatial Economics, 6, pp. 293-311, 2006.

[29] Taniguchi E. and Shimamoto H., Intelligent transportation system based dynamic vehicle routing and scheduling with variable travel times. Transportation Research, 12, pp. 235-250, 2004.

[30] Derekenaris G., Garofalakis J., Makris C., Prentzas J., Sioutas S. and Tsakalidis A., Integrating GIS, GPS and GSM technologies for the effective management of ambulances. Computers, Environment and Urban Systems, 25, pp. 267-278, 2001.

[31] Hardy M. and Wunderlich K., Evacuation Management Operations (EMO) modeling assessment: transportation modeling inventory, 2007. Source: http://www.its.dot.gov/its_publicsafety/emo/index.htm (last access: $12 / 01 / 12)$ 
[32] Hobeika A.G. and Jamei B., MASSVAC: a model for calculating evacuation times under natural disaster. Emergency Planning, Simulation Series, 15, pp. 23-28, 1985.

[33] Wallace W.A and De Balogh F., Decision support systems for disaster management. Public administration review, 1985.

[34] Theodoulou G. and Wolshon B., Alternative methods to increase the effectiveness of freeway contraflow evacuation. Transportation Research Record, 1865, pp. 48-56, 2004.

[35] Shiomi, Y., Seto, Y. and Uno, N., A medical facilities location model considering road network vulnerability and accessibility. Transportation Research Board 90, 2011.

[36] Hamza-Lup G.L., Hua K.A. and Peng R., Leveraging e-transportation in real- time traffic evacuation management. Electronic commerce research and applications, 6 (4), pp. 413-424, 2007.

[37] Tufekci S., An integrated emergency management decision support system for hurricane emergency. Safety Science, 20, pp. 39-48, 1995.

[38] Levy J.K. and Taji K., Group decision support for hazards planning and emergency management: a group analytic network process (GANP) approach. Mathematical and Computer Modelling, 46, pp. 906-917, 2007.

[39] Zhang Z. and Li Q., An open urban emergency decision support system. The International Archives of the Photogrammetry, Remote Sensing and Spatial Information Sciences, XXXVII (B4) pp. 1123-1127, 2008.

[40] Fernández L. J. E., de Cea J. and Valverde G. G., Effect of advanced traveler information systems and road pricing in a network with nonrecurrent congestion. Transportation Research A, 43, pp. 481-499, 2009.

[41] Maier-Speredellozzi V., Wang J.H., Collyer C. and Thomas N., Disseminating information with variable message signs during natural or human caused disasters. Source: http://www.ectri.org/YRS07/Papiers /Session-15/Mayer-Speredelozi.pdf (Last access: 01/12/11).

[42] Pel A.J., Hoogendoorn S.P. and Bliemer M.C.J., Evacuation modeling including traveler information and compliance behavior. Procedia engineering, 3, pp. 101-111, 2010.

[43] Emmerink R.H.M., Axhausen K.W., Ijkamp P. and Rietveld P., The potential of information provision in a simulated road transport network with non-recurrent congestion. Transportation Research C, 3(5), pp. 293309, 1995.

[44] Russo F. and Vitetta A., Reverse assignment: Calibrating link cost functions and updating demand from traffic counts and time measurements. Inverse Problems in Science and Engineering, Volume 19, Issue 7, pp. 921950, 2011.

[45] Russo F. and Vitetta A., An assignment model with modified Logit, which obviates enumeration and overlapping problems. Transportation, Volume 30, Issue 2, pp. 177-201, 2003.

[46] Huang H.J. and Li Z.C., A multiclass, multicriteria logit-based traffic equilibrium assignment model under ATIS. European Journal of Operational Research, 176, pp. 1464-1477, 2007. 
[47] Robinson M.R. and Khattak A., Traffic information source selection and use in emergency situations. Proc. of Transportation Research Board 90, 2011.

[48] Wang T. and Li Y., Research on the method of dynamic emergency rescue vehicle routing based on real time information. Proceedings of International conference on e-business intelligence, Atlantis Press, pp. 105-112, 2010.

[49] Beynon-Davies P., Human error and information systems failure: the case of the London ambulance service computer-aided despatch system project. Interacting with Computers, 11, pp. 699-720, 1999.

[50] Dixon C.S. and Haas R., GNSS user requirement in emergency management. Proc. of RIN NAV '08 Conference, 2008. 